\section{Public health and research funding for childhood neurodevelopmental disorders in Sub-Saharan Africa: a time to balance priorities}

\author{
Muideen O. Bakare, ${ }^{1}$ Kerim M. Munir, \\ Mashudat A. Bello-Mojeed ${ }^{3}$ \\ ${ }^{1}$ Child and Adolescent Unit, Federal \\ Neuropsychiatric Hospital, Enugu, \\ Nigeria; ${ }^{2}$ Division of Developmental \\ Medicine, Boston Children's Hospital, \\ Boston, MA, USA; ${ }^{3}$ Child and Adolescent \\ Unit, Federal Neuropsychiatric Hospital, \\ Lagos, Nigeria
}

\section{Abstract}

Sub-Saharan African (SSA) population consists of about $45 \%$ children, while in Europe and North America children population is 10$15 \%$. Lately, attention has been directed at mitigating childhood infectious and communicable diseases to reduce under-five mortality. As the under-five mortality index in Sub-Saharan Africa has relatively improved over the last two decades, more Sub-Saharan African children are surviving beyond the age of five and, apparently, a sizeable percentage of this population would be living with one or more childhood neurodevelopmental disorders (NDD). The distribution of child mental health service resources across the world is unequal. This manifests in the treatment gap of major childhood onset mental health problems in SSA, with the gap being more pronounced for childhood NDD. It is important to balance the public health focus and research funding priorities in Sub-Saharan Africa. We urgently need to define the burden of childhood NDD in the region for healthcare planning and policy formulation.

\section{Introduction}

Public health and research funding in SubSaharan Africa (SSA) has largely focused on communicable diseases, with less attention being paid to non-communicable diseases among the latter category NDD in children have a special place as they affect a major segment of the population. ${ }^{1}$ This linkage in disproportionate increase in developmental delays and intellectual disabilities was once termed as new morbidity and was one of the impetuses for the emergence of the special needs movement in the US. ${ }^{2}$ Many of these children are showing declines in cognitive functioning, delays in language skills, as well as poor motor and social skills. There is a need for urgent research to identify nodal points for early intervention. $^{2}$ One possible explanation for the current lack of public health attention to childhood NDD in SSA may be due to lack of human resource capacities for evaluation and interventions for children with complex NDD. Capacity building in terms of public health and clinical services as well as provision of research funding in this area are of paramount importance at the present time.

Neurodevelopmental disorders (NDD) are group of disorders arising from impairments in the developing brain and/or the central nervous system. They are considered neurodevelopmental in that by definition they originate during the developmental period, that is, during the prenatal, ante-natal, post-natal, infancy and early childhood periods. The disorders have varying degrees of associated burden on children, their families and their communities and almost always require multi-faceted services to address special educational, health care, social inclusion and rehabilitation needs. The NDD include intellectual developmental disorders with known genetic or metabolic etiologies, traumatic or congenital brain injuries including conditions such as cerebral palsy, as well as such prenatal exposures such as fetal alcohol syndrome, and disorders of social relatedness such as autism spectrum disorders (ASD).

Among the NDD of childhood, in particular, ASD has received great deal of attention in the US and Europe in the past decade. ASD comprise a group of complex, lifelong, disorders that are now usually identifiable prior to 3 years of age. ASD is characterized by qualitative impairments in reciprocal social interaction, impairments in verbal and non-verbal communication skills and a restricted pattern of interest or behavior (WHO, 1992; APA, 1994). ${ }^{3,4}$

In SSA, children under the age of 15 years on average consist about $36.5 \%$ (Ghana) to $50 \%$ (Uganda) of the overall population depending on country concerned. ${ }^{5}$ These figures contrast with under 15 -year child population distribution in Ireland (21\%), United States (20\%), UK (17.3\%), Netherlands (17\%), Sweden (15.4\%), Greece (14.2\%), and Germany (13.3\%). Europe and North America comprise about 15 to 20 percent of the total population (Figure 1). ${ }^{5}$ In SSA, the number of children that will go on to lead productive lives will therefore have an important impact not only in term of the quality of life of their own and their families, but will be of critical importance for sustaining the economic and political development of the region.

In view of the differences in health care priorities globally, public health and research
Correspondence: Muideen 0. Bakare, Child and Adolescent Unit, Federal Neuropsychiatric Hospital, Chime Avenue, Enugu, Nigeria.

Tel./Fax: +234.703.097.0079.

E-mail: mobakare2000@yahoo.com

Key words: public health, childhood neurodevelopmental disorders, Sub-Saharan Africa.

Acknowledgements: this work was partly supported by Fogarty International Center/NIH grants TW005807 and TW009248 (KMM).

Contributions: all authors contributed to the conception of the idea behind this article and were involved in revising the manuscript. MOB wrote the initial draft of the manuscript. All authors read and approved the final draft of the manuscript.

Received for publication: 10 April 2013.

Revision received: 4 July 2013.

Accepted for publication: 14 July 2013.

This work is licensed under a Creative Commons Attribution 3.0 License (by-nc 3.0).

CCopyright M.O. Bakare et al., 2014

Licensee PAGEPress, Italy

Healthcare in Low-resource Settings 2014; 2:1559 doi:10.4081/hls.2014.1559

funding policies in SSA had, to date, focused overwhelmingly on childhood communicable infectious diseases (e.g. pneumonia, diarrhea, malaria, HIV/AIDS) targeted towards reducing the rate of under-five mortality in the region in keeping with the Millennium Development Goals (MDG). ${ }^{6}$

\section{Declining under-five mortality in Sub-Saharan Africa}

Over the last decades in SSA, efforts had been directed through multiplex public health and research policies to curtail the communicable diseases that contributed greatly to under-five morbidity and mortality. These efforts are yielding important fruits based on the present indicators of under-five mortality over the past two decades in the region. ${ }^{7}$ The efforts had been moving the SSA region closer to achieving reduction in child mortality rate as part of the MDG 4 (Figure 2).

SSA region had achieved on the average about 30 percent reduction in under-five mortality rate between the period of 1990 and 2010 , it had also achieved double in its average rate of reduction from 1.2 percent a year between the period of 1990 and 2000 compared to 2.4 percent a year between the period of 2000 and 2010 (Figure 3). ${ }^{7}$ In absolute terms, 
four countries in SSA region had achieved the greatest reduction world-wide with up to 60 percent reduction in under five mortality rate over the last two decades and more than 4.5 percent yearly rate reduction on the average between the period of 1990 and 2010. These SSA countries included Malawi, Liberia, Niger and Sierra Leone. ${ }^{?}$

\section{Relative neglect of childhood neurodevelopmental disorders within the Sub-Saharan Africa public health framework}

While understandably communicable diseases and the attendant under-five mortality rate might have been great challenge to SSA region, current evidence suggests that there are ongoing substantial improvements in these indices. ${ }^{7}$ Going by the earlier observation that onset of symptoms of ASD and other NDD often coincide with the period of underfive morbidity and mortality in SSA children, ${ }^{8,9}$ it is logical to think that with the improvement in under-five mortality index in the region, the prevalence of ASD and other childhood NDD will be on the increase and deserve a greater level of public health significance. Ironically however, ASD and other childhood NDD have not been given adequate attention in the SSA region in terms of public health focus and research funding. ${ }^{10,11}$ Likewise, the WHO mhGAP has not emphasized the importance of the childhood NDD, focusing on adulthood disorders such as depression, national policies and rights of people with mental health conditions (where childhood NDD has a minor part, despite the disproportionate population numbers). To date, there has been no large scale epidemiological study of children to define the magnitude of the problem of ASD and other childhood NDD in the SSA region, which can be used for the purpose of planning and policy formulation in the region. ${ }^{10-13}$

\section{Autism spectrum disorders and other neurodevelopmental dis- orders in Africa}

It is well established that symptoms of ASD occur among African children contrary to earlier notions. ${ }^{14}$ Despite worldwide reports of increase prevalence of ASD, there is no large scale community based epidemiological data on ASD in Africa. A recent study on ASD among African children with intellectual and NDD suggests an increase in prevalence of ASD in Africa. ${ }^{13}$ There is a need for community based epidemiological study of ASD in Africa to confirm this trend. The onset of ASD symptoms among African children coincide with the period of less than five (and often 3) years of age that is characterized by vulnerabilities of African children to physical illness and infectious diseases associated with neurological consequences. ${ }^{15}$ There is an over-representation of non-verbal cases of ASD among African children presenting to orthodox medical facilities. ${ }^{16}$ The lack of or limited expressive language ability could be related to late interventions, resulting from late presentation and identification of ASD among African children. ${ }^{16}$ In Africa, it has been observed that ASD is rarely diagnosed exclusively of intellectual disabilities and there is a wide gap between age of onset of symptoms and diagnosis of ASD in Africa. ${ }^{10}$ Therefore identification and diagnosis of ASD has been observed to be late among African children. ${ }^{12}$ Possible factors identified from the literature that are associated with late identification of ASD in Africa include: poor knowledge and awareness about ASD; cultural beliefs and practices; tortuous pathway to care/ help-seeking behavior; inadequate number of trained personnel; inadequate healthcare facilities. ${ }^{16}$ There is scarcity of intervention programs for children with ASD and other NDD in Africa. The few available services are very expensive with huge unaffordable cost to most of the parents of affected children. ${ }^{12}$

\section{The changing paradigm}

In a review of pattern of funding for health in Africa between the year 2000 and 2002, communicable diseases have received the majority of funding, $52 \%$. This is followed by Nutrition and Food Security that has ranked second in funding, 28\%. Child and Adolescent Health and Survival, and Women's Health and Rights collectively received just about $3 \%$ of funding for this period. ${ }^{17}$ The present pattern of public health funding in Africa may need to be reviewed because of the changing paradigm outlined in this report. We need to move to at least an equal emphasis on non-communicable disorders affecting children that now survive well beyond 5 years of age and likely to suffer lifespan effects with ensuing long term economic and societal gains.

As noted earlier, the onset of ASD symptoms among African children coincide with the period of less than five years that is characterized by vulnerabilities of African children to physical illness as well as infectious diseases associated with neurological consequences that effect brain development ${ }^{10}$ Recent evidence suggests that with improvement in the index of under-five mortality in SSA and gradual progress being made towards achieving MDG
4, more children would be surviving beyond the age of five years and sizeable percentage of this population would be experiencing the burden of living with one or more NDD. ${ }^{10,18}$ The pertinent question is Is the SSA region getting braze-up for this challenges? The answer at this time is a resounding No.

\section{The way forward}

There is unequal distribution of mental health service resources across the world, which has manifested in a mental health treatment gap (mhGAP) which itself shows a specific imbalance for identification and treatment of major childhood mental disorders in SSA. ${ }^{1920}$ The unequal distribution of child men-

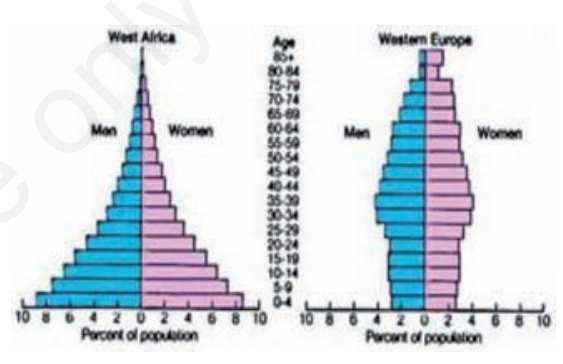

Figure 1. Contrast in West Africa and Western Europe population pyramids showing children population distribution.

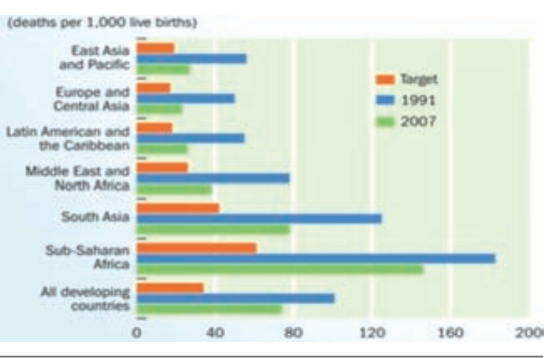

Figure 2. Though slowly, Sub-Saharan Africa is making progress towards reduction in under-five mortality and achieving the Millennium Development Goal 4.

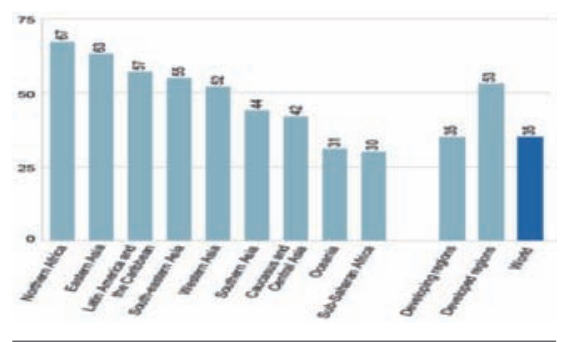

Figure 3. Many regions globally have reduced the under-five mortality rates by at least 50 percent between 1990 and 2010, with Sub-Saharan Africa achieving an average of $30 \%$ reduction. 
tal health service resources as it affects countries in SSA is more pronounced for childhood NDD. ${ }^{21}$ In line with MDG 6 which aims at achieving the objective of combating HIV/AIDS, malaria, and other diseases, research in the area of childhood NDD in SSA region is highly justified. Furthermore, despite a disproportionately lower percentage of children in the general population the research on childhood NDD, and in particular, research on ASD, in Europe and North America has seen a remarkable surge. This also needs to be justified as an urgent policy perspective in SSA. There is a distributive ethical need to redress the imbalance that is reflected by the much larger population of children in SSA compared to that in Europe and North America. ${ }^{22}$

\section{Conclusions}

The right time is now to conduct large scale epidemiological studies on ASD and other childhood NDD among SSA children. This is justified for the purpose of planning and policy formulation in the region that is urgently needed to address the burden of the problem on children that are surviving well beyond age 5 and to reduce the stigma upon families and communities at large. These objectives cannot be achieved without reviewing the present public health and research funding policies in the region. Finally, there is a need for major international NGOs to emphasize research in childhood NDD in the SSA context. Such a prioritization needs also to be reflected in the policies of the WHO Mental Health and Substance Abuse Department, as well as early childhood development policies of UNICEF, among others, to begin to understand the complex neurodevelopmental conditions affecting children's cognitive and social development. This needs to be achieved within a mental health paradigm, rather than a fantasy that somehow mental health does not exist and public health is all about prenatal care, maternal support, nutrition, immunization and prevention of communicable diseases. In order to effect lasting influences across the lifespan we need to prepare earlier on in the lifecycle for the identification of complex NDD and to provide cost effective early educational and behavioral interventions, as well as parent and teacher training strategies, social inclusion, and community empowerment. This is the time to balance priorities in public health and research funding, emphasizing childhood NDD in SSA.

\section{References}

1. Maher D, Ford N, Uwin N. Priorities for developing countries in the global response to non-communicable diseases. Global Health 2012;8:8-14.

2. Borkowski JG, Whitman TL, Passino AW, et al. Unraveling the "new morbidity": adolescent parenting and developmental delays. Int Rev Res Ment Ret 1992;18:15996.

3. WHO. International classification of diseases. 10th ed. Geneva: World Health Organization ed.; 1992.

4. APA. Diagnostic and statistical manual of mental disorders. 4th ed. Washington DC: American Psychiatric Association ed.; 1994.

5. UNDP. World development report. United Nations Development Programme ed; 2004. Available from: http:/hdr.undp.org/ en/media/hdr04_complete.pdf

6. Baingana FK, Bos ER. Changing pattern of diseases and mortality in Sub-Saharan Africa: an overview. In: Jamison DT, Feachem RG, Makogba MW, eds. Disease and mortality in Sub-Saharan Africa. 2nd ed. Washington DC: World Bank ed.; 2006.

7. You D, Wardlaw T. United Nations interagency group for child mortality estimation (2011 report): levels and trends in child mortality. Available from: http://resourcecentre.savethechildren.se/c ontent/library/documents/levels-trendschild-mortality-2011-report

8. Lotter V. Cross cultural perspectives on childhood autism. J Trop Pediatrics 1980; 26:131-3.

9. Mankoski RE, Collins M, Ndosi NK, et al. Etiologies of autism in a case-series from Tanzania. J Autism Dev Disord 2006; 36:1039-51.

10. Kauchali S, Davidson LL. Commentary: the epidemiology of neurodevelopmental disorders in Sub-Saharan Africa: moving forward to understand the health and psychosocial needs of children, families, and communities. Int J Epidemiol 2006;35:68990.

11. Idro R, Newton C, Kiguli S, KakoozaMwesige A. Child neurology practice and neurological disorders in East Africa. J Child Neurol 2010;25:518-24.

12. Bakare MO, Munir KM. Autism spectrum disorders in Africa. In: Mohammad-Reza M, ed. A comprehensive book on autism spectrum disorders. InTech, 2011. Rijeka: InTech; 2011. pp 183-95. Available from: http://www.intechopen.com/books/a-comprehensive-book-on-autism-spectrum-disorders/autism-spectrum-disorders-inafrica

13. Fuentes J, Bakare M, Munir K, et al. Autism spectrum disorders. In: Rey JM, ed. IACAPAP e-textbook of child and adolescent mental health. Geneva: International Association for Child and Adolescent Psychiatry and Allied Professions; 2012. pp C.2 1-27. Available from: http://iacapap.org/wp-content/uploads/C.2AUTISM-SPECTRUM-072012.pdf

14. Sanua VD. Is infantile autism a universal phenomenon? An open question. Int J Soc Psychiatr 1984;30:163-77.

15. Bakare M0, Ebigbo PO, Ubochi VN. Prevalence of autism spectrum disorders among Nigerian children with intellectual disability: a stopgap assessment. J Health Care Poor U 2012:23:513-8.

16. Bakare MO, Munir KM. Excess of non-verbal cases of autism spectrum disorders presenting to orthodox practice in Africa: a trend possibly resulting from late diagnosis and intervention. SAJP-S Afr J Psychi 2011:17:118-20.

17. Africa Grantmakers' Affinity Group. Funding for health in Africa: mapping the U.S Foundation Landscape 2000-2002. Washington, DC: The Tides Center/Africa Grantmakers' Affinity Group ed.; 2004. Available from: http://www.africagrantmakers.org/pdf/HIA.pdf

18. Mung'ala-Odera V, Meehan R, Njuguna P, et al. Prevalence and risk factors of neurological disability and impairment in children living in rural Kenya. Int J Epidemiol 2006;35:683-8.

19. Saraceno B, Dua T. Global mental health: the role of psychiatry. Eur Arch Psy Clin N 2009;259(Suppl.2):S109-17.

20. Omigbodun 0. Developing child mental health services in resource poor countries. Int Rev Psychiatr 2008;20:225-35.

21. Njenga F. Autism in Africa: a challenge in the management of an important disorder. Paper presented at the 13th IASSID World Congress Cape Town, South Africa, August 2008.

22. Yan EG, Munir K. Regulatory and ethical principles in research involving children and individuals with developmental disabilities. Ethics Behav 2004;14:31-49. 